\title{
Impact of Humoral Immune Response and Absorption Effect on Dynamics of Dengue Virus
}

\author{
S.D. Perera, M.Sc. \\ S.S.N. Perera, PhD
}

Research and Development Center for Mathematical Modeling, Faculty of

Science, University of Colombo, Sri Lanka

doi: 10.19044/esj.2017.v13n12p157 URL:http://dx.doi.org/10.19044/esj.2017.v13n12p157

\begin{abstract}
Dengue infection represents a global threat causing 50-100 million infections per year and placing half of the world's population at risk. Even though how infection is controlled and cured rather remains a mystery, antibodies are thought to play a major role in clearing the virus. In this paper, we study the dynamics of dengue virus with humoral immune response and absorption effect. The proposed model incorporates a time delay in production of antibodies. The basic reproduction number $\mathrm{R}_{0}$ is computed and a detailed stability analysis is done. It was found that the model has 3 steady states, namely, infection free equilibrium, no immune equilibrium and the endemic equilibrium. Conditions for $\mathrm{R}_{0}$ were developed for the local stability of these 3 equilibrium states. The global stability was studied using appropriate Lyapunov function and LaSalle's invariance principle. We then established a condition for which the endemic equilibrium point is globally asymptotically stable. Also it was observed that the virus count goes to negligible levels within 7-14 days after the onset of symptoms.
\end{abstract}

Keywords: Dengue, humoral immune response, absorption effect, basic reproduction number, stability

\section{Introduction}

Dengue continues to be a major public health concern in the world causing 50-100 million infections per year and placing more than two billion people at risk mainly in the tropical and subtropical regions of Africa, Asia and South America. (WHO-VMI Dengue Vaccine Modeling Group, 2012). The dengue virus is transmitted to humans primarily by the bite of infected female mosquito Aedes aegypti. The DENV complex consists of 4 related but distinct viruses designated as serotypes, DENV-1, DENV-2, DENV-3 and DENV-4. Infection with one dengue serotype may provide lifelong 
immunity to that serotype and a short term cross-protective immunity with respect to others (Rodrigues, Monteiro \& Torres, 2013).

Majority of individuals who experience dengue infection have asymptotic infection or mild disease known as Dengue Fever (DF) which usually resolves within 7-14 days after onset of fever along with symptoms such as headaches, muscle or joint pain, rash and so on by a complex immune response process (Gujarati \& Ambika, 2014; Nuraini, Tasman, Soewono \& Sidarto, 2009; PAHO, 1994). Only a few would proceed to severe dengue, Dengue Hemorrhagic Fever (DHF) and Dengue Shock Syndrome (DSS). Without proper monitoring and immediate treatment DHF and DSS can be fatal.

Though there is no effective treatment for dengue, understanding within host viral dynamics will help in developing interventions against dengue. Objective of this paper is to understand how immune process is involved in clearing the dengue infection. The human immune system is made up of two parts, the innate immune system and the adaptive immune system which produce antibody-secreting $\mathrm{B}$ cells (humoral immune response) and cytotoxic $\mathrm{T}$ cells (cell-mediated immune response) both of which are responsible to clear the infection and provide life-long immunity against a pathogen (Host Response to the Dengue Virus; Nikin-Beers \& Ciupe, 2015; Wahala and de Silva, 2011; Janeway CA et al., 2001). Since antibodies play a more prominent role in clearing the dengue infection, in this study we consider only the antibody mediated immune response. The schematic representation of the human immune system is shown in Fig.1.

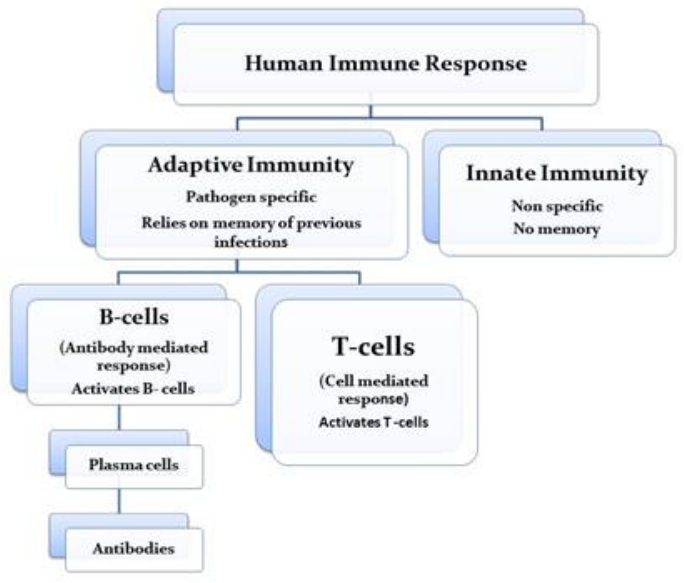

Fig. 1: human immune system

Mathematical models on dengue epidemiology have been widely studied. (Derouich \& Boutayeb, 2006; Guzmán \& Kourí, 2004; Summer, 2010). 
However models on within host dengue viral dynamics has not been given much attention. Most of the existing models focus on virus dynamics without considering the absorption of pathogens into uninfected cells (Gujarati \& Ambika, 2014; Nikin-Beers \& Ciupe, 2015; Rotem \& Koelle). As a result of absorption, pathogen density in the blood reduces with time. Thus in this study, we incorporate the loss of virus due to absorption effect.

In section 2, a model with humoral immune response and absorption effect is formulated. In Section 3 a detail mathematical analysis of the model is done. Conclusion is presented in Section 4.

\section{The Model}

We modify the model presented in Nuraini et al. (2009) by introducing additional terms which describes the humoral immune response for within host primary dengue infection.

The model is given by the following nonlinear system of equations

$$
\begin{aligned}
& \frac{d S}{d t}=\mu-\alpha S-a S V, \\
& \frac{d I}{d t}=a S V-\beta I, \\
& \frac{d V}{d t}=k I-\gamma V-a S V-p V Z, \\
& \frac{d Z}{d t}=H(t-\tau) \eta+d V Z-\delta Z,
\end{aligned}
$$

where $S$-healthy cells (monocytes, macrophages, dendritic cells, hepatocytes or mast cells), $I$-infected cells, $V$-Dengue virus particles and $Z$-neutralizing antibodies. The description of parameters is given in Table 1 .

The production of antibodies is a multi-step complex process. Once the adaptive immune response starts fighting the dengue infection, B-cells in human body come into contact with virus and they become activated. These activated B-cells proliferate and mature into plasma cells. These plasma cells produce antibodies which circulate throughout the body binding to the virus and making them non-infectious (Gujarati \& Ambika, 2014; Nikin-Beers \& Ciupe, 2015). The delay in antibody production, $\tau$, is significant and is introduced through a Heaviside step function, $H$. A numerical simulation of the equations of the model is given in Fig.2. Here we have assumed that the time delay in antibody production is 3 days. 

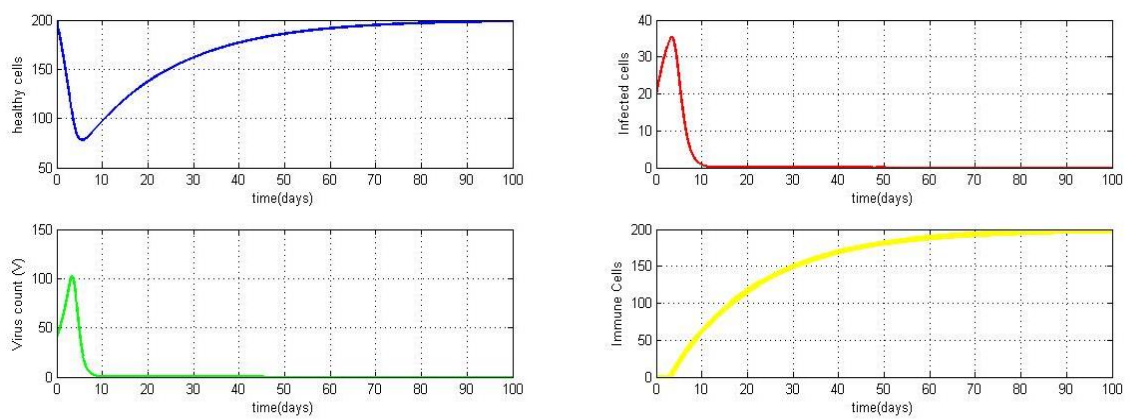

Fig 2: Viral dynamics with absorption for $\tau=3$. The parameter values used are $\mu=10, \alpha=0.05$, $\mathrm{a}=0.003, \beta=0.9, \gamma=0.1, \mathrm{p}=0.01, \delta=0.05, \mathrm{~d}=0.001, \mathrm{k}=2, \eta=10$. Initial condition

$(\mathrm{S}, \mathrm{I}, \mathrm{V}, \mathrm{Z})=(200,20,40,0)$

By introducing the transformation $\bar{Z}=Z-\frac{\eta}{\delta}$ as described in Nuraini et al. (2009) and replacing $\bar{Z}$ with $Z$, the system reads as

$$
\begin{aligned}
& \frac{d S}{d t}=\mu-\alpha S-a S V, \\
& \frac{d I}{d t}=a S V-\beta I, \\
& \frac{d V}{d t}=k I-f V-a S V-p V Z, \\
& \frac{d Z}{d t}=H \frac{d \eta}{\delta} V+d V Z-\delta Z,
\end{aligned}
$$

\begin{tabular}{|c|c|}
\hline $\begin{array}{c}\text { Parameter } \\
\text { Symbol }\end{array}$ & Parameter Description \\
\hline$\mu$ & Production rate of healthy cells \\
\hline$\alpha$ & Death rate of healthy cells \\
\hline$a$ & $\begin{array}{l}\text { Rate at which healthy cells are converted to infected cells due to their } \\
\text { interaction with virus particles }\end{array}$ \\
\hline$\beta$ & Death rate of infected cells \\
\hline$k$ & Burst rate of virus particles \\
\hline$\gamma$ & Rate at which virus particles degrade \\
\hline$p$ & Rate at which virus particles are neutralized by antibodies \\
\hline$\eta$ & production rate of immune cells \\
\hline$d$ & Rate at which immune cells are stimulated by virus particles \\
\hline$\delta$ & Death rate of immune cells \\
\hline
\end{tabular}

where

$$
f=\gamma+\frac{p \eta}{\delta} .
$$

Table 1: Description of the parameters 


\section{Mathematical Analysis of the model:}

\section{Basic Reproduction Number}

For within-host dengue viral dynamics, the basic reproduction number $R_{0}$ is defined as the average number of secondary infections generated by a single infected cell placed in an uninfected cell population (Nikin-Beers \& Ciupe, 2015; Nowak \& May, 2000).

By using the next generation method, we obtain the following basic reproduction number for model (2)

$$
R_{0}=\frac{a \mu k}{\beta(\alpha f+a \mu)} .
$$

For a similar model, without absorption effect (without the term $a S V$ in the $3^{\text {rd }}$ equation of model (2)), the basic reproduction number was computed and is given by,

$$
R_{0 i}=\frac{a \mu k}{\alpha \beta f} .
$$

For model (2), the basic reproduction number is less than that of a model without absorption effect.

\section{Equilibria of the model}

We are interested in looking at parameter values that leads to infection free steady sates and infectious states. For this we do a detailed stability analysis of the equilibrium points of model (2).

Considering the steady states of system (2), the equilibrium points can be obtained and are given by

$$
\begin{aligned}
S^{*} & =\frac{\mu}{\alpha+a V^{*}} \\
I^{*} & =\frac{a S^{*} V^{*}}{\beta} \\
Z^{*} & =\frac{d \eta V^{*}}{\delta\left(\delta-d V^{*}\right)}
\end{aligned}
$$

By substituting equations (4) \& (6) in the $3^{\text {rd }}$ equation of model (2) and solving for its steady state we obtain, 


$$
\begin{aligned}
& P_{3} V^{* 3}+P_{2} V^{* 2}+P_{1} V^{*}=0 \\
& V^{*}\left(P_{3} V^{* 2}+P_{2} V^{*}+P_{1}\right)=0
\end{aligned}
$$

Thus

$$
V^{*}=0 \quad \text { or } \quad P_{3} V^{* 2}+P_{2} V^{*}+P_{1}=0
$$

where

$$
\begin{aligned}
& P_{3}=a \beta \delta f d-a \beta \eta p d \\
& P_{2}=\delta \beta d(\alpha f+a \mu)\left[\left(1-R_{0}\right)-\frac{a \delta^{2} f+\alpha \eta p d}{\delta d(\alpha f+a \mu)}\right] \\
& P_{1}=\beta \delta^{2}(\alpha f+a \mu)\left(R_{0}-1\right)
\end{aligned}
$$

Equation (7) has one solution $V^{*}=0$ and another $V^{*} \neq 0$ solution.

\section{Local Stability}

Next we analyze the stability of the infection free equilibrium $\left(V^{*}=0\right)$ and endemic equilibrium $\left(V^{*} \neq 0\right)$. For this we must first linearize the model about its equilibrium points and the corresponding Jacobian matrix is given by

$$
J=\left[\begin{array}{cccc}
-\left(\alpha+a V^{*}\right) & 0 & -a S^{*} & 0 \\
a V^{*} & -\beta & a S^{*} & 0 \\
-a V^{*} & k & \left(-f-a S^{*}-p Z^{*}\right) & -p V^{*} \\
0 & 0 & \left(\frac{d \eta}{\delta}+d Z^{*}\right) & d V^{*}-\delta
\end{array}\right]
$$

The characteristic equation for $\mathrm{J}$ would take the form,

$$
G(\lambda)=\lambda^{4}+G_{3} \lambda^{3}+G_{2} \lambda^{2}+G_{1} \lambda+G_{0}=0 \text {. }
$$

The stability properties that were established are summarized in the following theorems.

\section{Infection free steady state}

\section{Theorem 1}

The disease free equilibrium $E_{1}=\left(\frac{\mu}{\alpha}, 0,0,0\right)$ is locally asymptotically stable if $R_{0}<1$. If $R_{0}>1$, the equilibrium $E_{1}$ is unstable. 


\section{Proof}

After substituting the equilibrium values for $S^{*}, I^{*}, Z^{*}$ obtained from equation (4), (5) and (6) and applying $V^{*}=0$ in (12), the characteristic equation for $\mathrm{J}$ can be written as

$G(\lambda)=(\alpha+\lambda)(\delta+\lambda)\left(\frac{\alpha \lambda^{2}+\alpha \beta f+a \beta \mu-a k \mu+\alpha \beta \lambda+\alpha f \lambda+a \mu \lambda}{\alpha}\right)=0$

where $\lambda$ is an eigenvalue.

This can be further simplified to

$G(\lambda)=(\alpha+\lambda)(\delta+\lambda)\left(\alpha \lambda^{2}+(\alpha(\beta+f)+a \mu) \lambda+\beta(\alpha f+a \mu)\left(1-R_{0}\right)\right)=0$

The eigenvalues of this equation are

$\lambda_{1}=-\alpha$

$\lambda_{2}=-\delta$

$\lambda_{3}=\frac{-(\alpha(\beta+f)+a \mu) \pm \sqrt{(\alpha(\beta+f)+a \mu)^{2}-4 \alpha \beta(\alpha f+a \mu)\left(1-R_{0}\right)}}{2 \alpha}$

For $R_{0}<1, G(\lambda)=0$ has all negative roots. Thus the equilibrium point $E_{1}$ is locally asymptotically stable.

If $R_{0}>1, G(\lambda)=0$ has 2 negative roots and one positive root. Thus the equilibrium point $E_{1}$ is unstable.
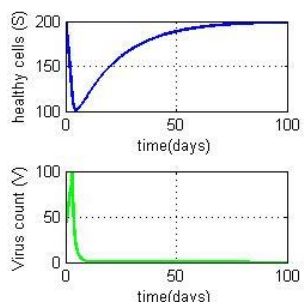

Fig 3(a): Infection free equilibrium for $\mathrm{R}_{0}<1$. The numerical values of the parameters used are $\mu=10, \alpha=0.05, \mathrm{a}=0.003$, $\beta=0.9, \gamma=0.1, p=0.01, \delta=0.05, d=0.001, k=2$, $\eta=10$.
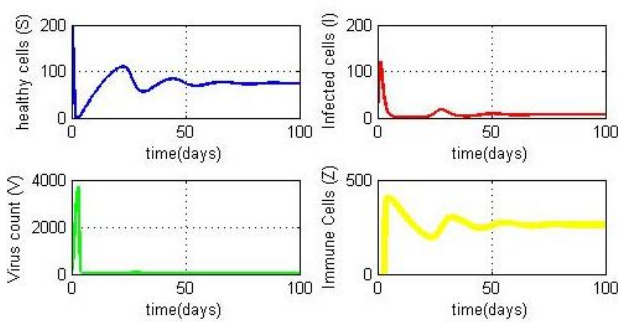

Fig 3(b): Infection free equilibrium for

$\mathrm{R}_{0}>1$. The numerical values of the parameters used are $\mu=10, \alpha=0.05, \mathrm{a}=0.003$, $\beta=0.9, \gamma=0.1, \mathrm{p}=0.01, \delta=0.05, \mathrm{~d}=0.001$, $\mathrm{k}=20, \eta=10$.

It is clear from Fig.3 (a) that for $R_{0}<1, S^{*}$ converges to $\frac{\mu}{\alpha}, I^{*}, V^{*}$ and $Z^{*}$ converges to 0. From Fig.3 (b) we can see that when $R_{0}>1$, the equilibrium becomes unstable. 


\section{Endemic Equilibriums}

The limiting condition where virus achieves its maximum due to the absence of linear response is $\frac{d \eta}{\delta}$. Thus the no immune equilibrium can be obtained in two ways either $d=0$ or $\eta=0$.

Case 1

Let $d=0$.

Then from equations (9), (10) and (11) we have, $P_{3}=0$

$P_{2}=-a \beta \delta^{2} f$

$P_{1}=a \mu k \delta^{2}-a \mu \beta \delta^{2}-\alpha \beta \delta^{2} f$

and from equation (8),

$$
V^{*}=\frac{-P_{1}}{P_{2}}=\frac{-a \mu k \delta^{2}+a \mu \beta \delta^{2}+\alpha \beta \delta^{2} f}{-a \beta \delta^{2} f}
$$

which can be simplified to

$$
V^{*}=\frac{a \mu k-\beta(a \mu+\alpha f)}{-a \beta \delta^{2} f}
$$

Thus we have the no immune endemic equilibrium corresponding to $\mathrm{d}=0$,

$$
E_{2}=\left(\frac{\beta f}{a(k-\beta)}, \frac{a \mu k-\beta(a \mu+\alpha f)}{a \beta(k-\beta)}, \frac{a \mu k-\beta(a \mu+\alpha f)}{a \beta f}, 0\right)
$$

The local stability property of $E_{2}$ is given by the following theorem.

\section{Theorem 2}

Let $d=0$. If $k-\beta>0$ and $R_{0}>1$, then the no immune equilibrium $E_{2}$ is locally asymptotically stable.

\section{Proof}

The characteristic equation corresponding to the equilibrium $E_{2}$ can be simplified to 


$$
\begin{gathered}
H(\lambda)=(\delta+\lambda)\left(\lambda^{3}+\left(\beta+\frac{f k}{k-\beta}+\frac{a \mu(k-\beta)}{\beta f}\right) \lambda^{2}+\left(\frac{a \mu(k-\beta)}{f}+\frac{a \mu k}{\beta}\right) \lambda+\right. \\
\left.\frac{\beta k(\alpha f+a \mu)}{k-\beta}\left(R_{0}-1\right)\right)=0
\end{gathered}
$$

The eigenvalues of equation (16) are

$$
\lambda_{1}=-\delta
$$

and the roots of

$$
\lambda^{3}+q_{2} \lambda^{2}+q_{1} \lambda+q_{0}=0
$$

where

$$
\begin{aligned}
& q_{2}=\beta+\frac{f k}{k-\beta}+\frac{a \mu(k-\beta)}{\beta f} \\
& q_{1}=\frac{a \mu(k-\beta)}{f}+a \mu\left(\frac{k-\beta}{\beta}\right)+\frac{\alpha \beta f}{k-\beta}+\frac{a \mu k^{2}}{\beta(k-\beta)} \\
& q_{0}=\beta(\alpha f+a \mu)\left(R_{0}-1\right)
\end{aligned}
$$

Since $k>\beta$ and $R_{0}>1, q_{0}, q_{1}$ and $q_{2}$ are all positive.

Also it can be proven that $q_{1} q_{2}>q_{0}$.

Thus by the Routh Hurwitz criterion, the roots of equation (17) has all negative real parts. Hence all roots of the characteristic equation (16) have negative real parts when $k>\beta$ and $R_{0}>1$. Thus $\mathrm{E}_{2}$ is locally asymptotically stable. Fig. 4 demonstrates this phenomenon.
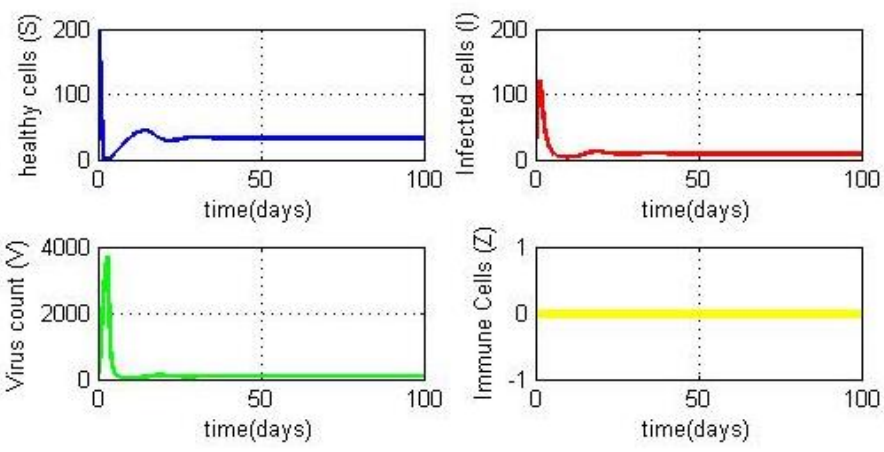

Fig 4: No immune equilibrium for $d=0$ and for $R_{0}>1$. The numerical values of the parameters used are $\mu=10, \alpha=0.05, a=0.003, \beta=0.9, \gamma=0.1, p=0.01, \delta=0.05, k=20, \eta=10$ and $\boldsymbol{\tau}=3$. 
Case 2

Let $\eta=0$.

Then from equations (9), (10) and (11) we have,

$P_{3}=a \beta \delta \gamma d$

$P_{2}=-a \mu k \delta d+a \mu \beta \delta d-a \beta \delta^{2} \gamma+\alpha \beta \gamma d$

$P_{1}=a \mu k \delta^{2}-a \mu \beta \delta^{2}-\alpha \beta \gamma \delta^{2}$

and by solving

$P_{3} V^{* 3}+P_{2} V^{* 2}+P_{1} V^{*}=0$

We get,

$V^{*}=0$, which always exists, $V^{*}=\frac{\delta}{d}$, which has no biological interest

and

$V^{*}=\frac{a \mu k-\beta(a \mu+\alpha \gamma)}{a \beta \gamma}$.

The no immune endemic equilibrium corresponding to $\eta=0$ is,

$E_{3}=\left(\frac{\beta \gamma}{a(k-\beta)}, \frac{a \mu k-\beta(a \mu+\alpha \gamma)}{a \beta(k-\beta)}, \frac{a \mu k-\beta(a \mu+\alpha \gamma)}{a \beta \gamma}, 0\right)$.

The stability property of $\mathrm{E}_{3}$ is summarized in Theorem 3 .

\section{Theorem 3}

Let $\eta=0$. If $k-\beta>0$ and $1<R_{0}<1+\frac{a \delta \gamma}{d(\alpha \gamma+a \mu)}$, then the no immune equilibrium $E_{3}$ is locally asymptotically stable. If $R_{0}>1+\frac{a \delta \gamma}{d(\alpha \gamma+a \mu)}$, it becomes unstable.

\section{Proof}

Local stability of the equilibrium point $\mathrm{E}_{3}$ is governed by the eigenvalues of the characteristic equation corresponding to $\mathrm{E}_{3}$.

One of the eigenvalues is $\lambda_{1}=-\delta+\frac{a d \mu(k-\beta)-\alpha \beta \gamma d}{a \beta \gamma}$.

If $\quad R_{0}<1+\frac{a \delta \gamma}{d(\alpha \gamma+a \mu)}$ 
$\frac{a \mu k}{\beta(\alpha \gamma+a \mu)}<1+\frac{a \delta \gamma}{d(\alpha \gamma+a \mu)}$

by rearranging we have,

$-\delta+\frac{a d \mu(k-\beta)-\alpha \beta \gamma d}{a \beta \gamma}<0$

Thus $\lambda_{1}<0$.

The other eigenvalues are the roots of

$\lambda^{3}+q_{2} \lambda^{2}+q_{1} \lambda+q_{0}=0$

where

$q_{2}=\beta+\frac{\gamma k}{k-\beta}+\frac{a \mu(k-\beta)}{\beta \gamma}$

$q_{1}=\frac{a \mu(k-\beta)}{\gamma}+a \mu\left(\frac{k-\beta}{\beta}\right)+\frac{\alpha \beta \gamma}{k-\beta}+\frac{a \mu k^{2}}{\beta(k-\beta)}$

$q_{0}=\beta(\alpha \gamma+a \mu)\left(R_{0}-1\right)$

Since $k>\beta$ and $R_{0}>1, q_{0}, q_{1}$ and $q_{2}$ are all positive.

Also it can be proven that $q_{1} q_{2}>q_{0}$.

Thus by the Routh Hurwitz criterion, the roots of equation (20) has all negative real parts.

Hence all eigenvalues have negative real parts. Thus $E_{3}$ is locally asymptotically stable.

If $R_{0}>1+\frac{a \delta \gamma}{d(\alpha \gamma+a \mu)}$

By rearranging we get,

$-\delta+\frac{a d \mu(k-\beta)-\alpha \beta \gamma d}{a \beta \gamma}>0$

Thus $\lambda_{1}>0$.

Therefore at least one eigenvalue has a positive real part hence $\mathrm{E}_{3}$ becomes unstable.

Further there exists another endemic equilibrium, $\mathrm{E}_{4}$, virus persistence in the presence of antibodies.

$E_{4}=(\hat{S}, \hat{I}, \hat{V}, \hat{Z})=\left(\frac{\mu d}{\alpha d+a \delta}, \frac{a \delta \hat{S}}{\beta d}, \frac{\delta}{d},\left(\frac{a \hat{S}}{p}\left(\frac{k}{\beta}-1\right)-\frac{\gamma}{p}\right)\right)$

$\mathrm{E}_{4}$ exists provided that, 
$\frac{a \hat{S}}{p}\left(\frac{k}{\beta}-1\right)-\frac{\gamma}{p}>0 \quad$ where $\quad \hat{S}=\frac{\mu d}{\alpha d+a \delta}$

which implies

$\hat{S}>\frac{\beta \gamma}{a(k-\beta)}$

Hence we have the results in theorem 4.

\section{Theorem 4}

Let $\eta=0$ and $\hat{\mathrm{Z}} \neq 0$. If $k-\beta>0$ and $R_{0}>1+\frac{a \delta \gamma}{d(\alpha \gamma+a \mu)}$, then the equilibrium $E_{4}$ is locally asymptotically stable.

\section{Proof}

The characteristic equation at the equilibrium $E_{4}$ can be written as

$$
\begin{aligned}
&(\beta+\lambda)\left(\beta \lambda^{2}-\beta \delta \gamma-\hat{S} a \beta \delta+\hat{S} a \delta k\right)((\alpha+\lambda)+a \delta)+ \\
&(\alpha+\lambda) \hat{S} a k \lambda^{2}+\hat{S} a^{2} \delta(\beta+\lambda)(k-\beta) \quad=0
\end{aligned}
$$

It can be shown that all roots of equation (22) has negative real parts if $\hat{S}>\frac{\beta \gamma}{a(k-\beta)}$ and $k>\beta$.

Thus we have, $\frac{\mu d}{\alpha d+a \delta}>\frac{\beta \gamma}{a(k-\beta)}$

By rearranging,

$$
\frac{a \mu d k}{\beta d(\alpha \gamma+a \mu)}-\frac{a \beta \delta \gamma}{\beta d(\alpha \gamma+a \mu)}>1
$$

Hence $R_{0}>1+\frac{a \delta \gamma}{d(\alpha \gamma+a \mu)}$.

Numerically we can see this in Fig. (5). 

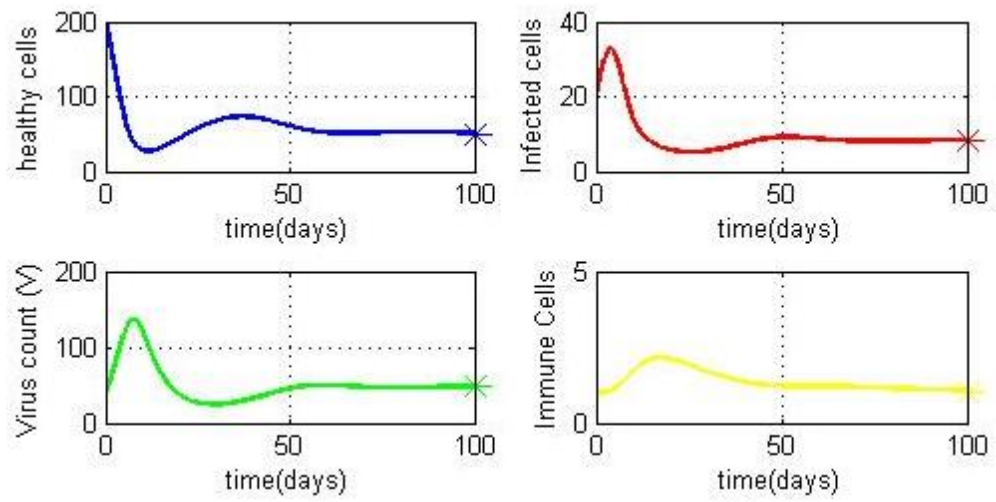

Fig 5: Endemic equilibrium. In this case $R_{0}>1+\frac{a \delta \gamma}{d(\alpha \gamma+a \mu)}$. The equilibrium points are marked with an ' $*$ '. We can see that $\hat{S}, \hat{I}, \hat{V}$ and $\hat{Z}$ converges to the equilibrium values. The numerical values of the parameters used are $\mu=10, \alpha=0.05$, $a=0.003, \beta=0.9, \gamma=0.1$, $\mathrm{p}=0.08, \delta=0.05, \mathrm{~d}=0.001, \mathrm{k}=2, \eta=0$ and $\boldsymbol{\tau}=3$.

\section{Global Stability}

\section{Theorem 5}

We may assume that the equilibrium point $E_{4}$ appears in the interior of the first quadrant. That is $R_{0}>1+\frac{a \delta \gamma}{d(\alpha \gamma+a \mu)}$. When $k>\beta\left(1+\frac{a \hat{V}}{\alpha}\right)$ holds, $W(S, I, V, Z)=A(S-\hat{S} \ln S)+k(I-\hat{I} \ln I)+B(V-\hat{V} \ln V)+E(Z-\hat{Z} \ln Z)$ is a continuously differentiable function, and the interior equilibrium $(\hat{S}, \hat{I}, \hat{V}, \hat{Z})$ is globally asymptotically stable in the interior of the $1^{\text {st }}$ quadrant.

\section{Proof}

Note that $E_{4}$ is obtained by letting $\eta=0$. Let

$W(S, I, V, Z)=A(S-\hat{S} \ln S)+k(I-\hat{I} \ln I)+B(V-\hat{V} \ln V)+E(Z-\hat{Z} \ln Z)$

Where $(\hat{S}, \hat{I}, \hat{V}, \hat{Z})$ is given by equation (21).

$$
\dot{W}=A\left(\dot{S}-\frac{\hat{S}}{S} \dot{S}\right)+k\left(\dot{I}-\frac{\hat{I}}{I} \dot{I}\right)+B\left(\dot{V}-\frac{\hat{V}}{V} \dot{V}\right)+E\left(\dot{Z}-\frac{\hat{Z}}{Z} \dot{Z}\right)
$$

After substituting for $(\dot{S}, \dot{I}, \dot{V}, \dot{Z})$ by equation (1) we obtain, 


$$
\begin{aligned}
\dot{W} & =A(\mu-\alpha S-a S V)-A \frac{\hat{S}}{S}(\mu-\alpha S-a S V)+k(a S V-\beta I)+B(k I-a S V-\gamma V-p V Z) \\
& +E(d V Z-\delta Z)-k \frac{\hat{I}}{I}(a S V-\beta I)-B \frac{\hat{V}}{V}(k I-a S V-\gamma V-p V Z)-E \frac{\hat{Z}}{Z}(d V Z-\delta Z)
\end{aligned}
$$

This can be further simplified to,

$$
\begin{aligned}
& \dot{W}=A(\mu-\alpha S)-A \frac{\hat{S}}{S}(\mu-\alpha S)+(k-B-A) a S V+(B-\beta) k I+(E d-B p) V z-E \delta Z \\
& +(A a \hat{S} V-B \gamma V)-k \frac{\hat{I}}{I}(a S V-\beta I)-B \frac{\hat{V}}{V}(k I-a S V-\gamma V-p V Z)-E \frac{\hat{Z}}{Z}(d V Z-\delta Z)
\end{aligned}
$$

Now we define $\mathrm{A}, \mathrm{B}$ and $\mathrm{E}$ by letting $k-B-A=0, B-\beta=0$ and $E d-p B=0$.

From these we get, $B=\beta, k-\beta=A, E=\frac{p \beta}{d}$.

From the second and third equations in (1), we obtain $\hat{I}=\frac{a \hat{S} \hat{V}}{\beta}$ and $k \hat{I}-a \hat{S} \hat{V}-\gamma \hat{V}-p \hat{V} \hat{Z}=0$

From these we can derive, $A a \hat{S} V-B \gamma V=(k-\beta) a \hat{S} V-\beta \gamma V=\beta p V \hat{Z}$.

after substituting $\frac{\delta}{d}=\hat{V}$ and equation (24) we can simplify equation (23) to

$$
\begin{aligned}
\dot{W}= & (k-\beta)(\mu-\alpha S)-(k-\beta) \frac{\hat{S}}{S}(\mu-\alpha S)-k \frac{\hat{I}}{I} a S V+k \beta \hat{I}-\beta k I \frac{\hat{V}}{V} \\
& +\gamma \beta \hat{V}+\beta a S \hat{V}+p \beta \hat{V} \hat{Z}
\end{aligned}
$$

Using $\mu=\alpha \hat{S}+a \hat{S} \hat{V}$ which follows from equation (1) we have,

$$
(k-\beta)\left(\mu-\alpha S-\frac{\hat{S}}{S} \mu+\alpha \hat{S}\right)=\alpha \hat{S}(k-\beta)\left(2-\frac{S}{\hat{S}}-\frac{\hat{S}}{S}\right)+(k-\beta)\left(a \hat{S} \hat{V}-\frac{a \hat{S}^{2} \hat{V}}{S}\right)
$$

From equation (1) we also have $\beta \hat{I}=a \hat{S} \hat{V}$ and $\mathrm{p} \hat{V} \hat{Z}=k \hat{I}-a \hat{S} \hat{V}-\gamma \hat{V}$.

Simplification of

$$
\begin{aligned}
& (k-\beta)\left(a \hat{S} \hat{V}-\frac{a \hat{S}^{2} \hat{V}}{S}\right)-k \frac{\hat{I}}{I} a S V+k \beta \hat{I}-\beta k I \frac{\hat{V}}{V}+\gamma \beta \hat{V}+\beta a S \hat{V}+p \beta \hat{V} \hat{Z} \\
& =k \beta \hat{I}\left(3-\frac{\hat{S}}{S}-\frac{\hat{V} I}{V \hat{I}}-\frac{\hat{I} S V}{I \hat{S} \hat{V}}\right)-a \beta \hat{S} \hat{V}\left(2-\frac{\hat{S}}{S}-\frac{S}{\hat{S}}\right)
\end{aligned}
$$

From equations (25), (26) and (27) we have 


$$
\begin{aligned}
& \dot{W}=(k-\beta)\left(\mu-\alpha S-\frac{\hat{S}}{S} \mu+\alpha \hat{S}\right)-k \frac{\hat{I}}{I} a S V+k \beta \hat{I}-\beta k I \frac{\hat{V}}{V}+\gamma \beta \hat{V}+\beta a S \hat{V}+p \beta \hat{V} \hat{Z} \\
& \dot{W}=\alpha \hat{S}(k-\beta)\left(2-\frac{S}{\hat{S}}-\frac{\hat{S}}{S}\right)+k \beta \hat{I}\left(3-\frac{\hat{S}}{S}-\frac{\hat{V} I}{V \hat{I}}-\frac{\hat{I} S V}{I \hat{S} \hat{V}}\right)-a \beta \hat{S} \hat{V}\left(2-\frac{\hat{S}}{S}-\frac{S}{\hat{S}}\right)
\end{aligned}
$$

By further simplification

$\dot{W}=\left(k-\beta\left(1+\frac{a \hat{V}}{\alpha}\right)\right) \alpha \hat{S}\left(2-\frac{S}{\hat{S}}-\frac{\hat{S}}{S}\right)+k \beta \hat{I}\left(3-\frac{\hat{S}}{S}-\frac{\hat{V I}}{V \hat{I}}-\frac{\hat{I} S V}{I \hat{S} \hat{V}}\right)$

Since $\alpha \hat{S}\left(2-\frac{S}{\hat{S}}-\frac{\hat{S}}{S}\right)=-\alpha \frac{(S-\hat{S})^{2}}{S}$ and

Since Arithmetic mean> Geometric mean,

$$
k \beta \hat{I}\left(3-\frac{\hat{S}}{S}-\frac{\hat{V I}}{V \hat{I}}-\frac{\hat{I} S V}{I \hat{S} \hat{V}}\right)>0
$$

From equations (28), (29) and (30) we can conclude that if $k-\beta\left(1+\frac{a \hat{V}}{\alpha}\right)>0$, then $\dot{W} \leq 0$.

Let $E=\left\{(S, I, V, Z) \in \mathfrak{R}_{+}^{4} \mid S, I, V, Z>0, \dot{V}=0\right\}$ is

$$
E=\left\{(\hat{S}, I, V, \hat{Z}) \mid \frac{I}{\hat{I}}=\frac{V}{\hat{V}}, I>0, V>0\right\}
$$

Let $M$ be the largest invariant set in $E$. If $M$ contains some point $(\hat{S}, I, V, \hat{Z})$ such that $V \neq \hat{V}$ then $\frac{d Z}{d t} \neq 0$. Thus a contradiction occurs.

Therefore $V=\hat{V}$ and $I=\hat{I}$. Hence by LaSalle's invariance principle, $\mathrm{E}_{4}$ is globally asymptotically stable in the interior of the $1^{\text {st }}$ quadrant.

\section{Conclusion}

A mathematical model with antibody mediated immune response and absorption effect was developed to study within host dengue virus dynamics. We assumed that there is a time delay in production of antibodies and at the time of infection antibodies are below detection levels. A detailed stability analysis of equilibriums were done and it was found that the model has 3 steady states, namely, the infection free equilibrium, no immune equilibrium and the virus persistence in the presence of antibodies equilibrium. By 
establishing the characteristic equation of the model at infection free equilibrium, it was observed that the infection free equilibrium is locally asymptotically stable if $\mathrm{R}_{0}<1$. The no immune equilibrium was obtained in two ways either by making the linear immune response $\eta=0$ or by making the nonlinear immune response $\mathrm{d}=0$. In the absence of immune response, the virus count would settle to some nonzero value making the infection endemic.

Local stability property of endemic equilibrium was derived. It was noted that if $R_{0}>1+\frac{a \delta \gamma}{d(\alpha \gamma+a \mu)}$, the endemic equilibrium is locally asymptotically stable. By introducing a suitable Lyapunov function and considering LaSalle's invariance principle, it was observed that the endemic equilibrium is globally asymptotically stable if $k>\beta\left(1+\frac{a \hat{V}}{\alpha}\right)$.

We found that the basic reproduction number for model (2) is less than that of a similar model without absorption effect. Thus it can be seen that absorption effect has a positive influence on virus clearance from the body than that of a model without absorption effect. Numerical simulation results provide evidence that in general the dengue virus gets cleared from the body within 7-14 days which is agreeable with clinical studies.

Further, the model can be improved by including a continuous function for the time delay. Also we can analyze the effects on the model by changing the delay in the antibody production.

\section{Acknowledgement}

The authours would like to thank University of Colombo grant no: $\mathrm{AP} / 3 / 2 / 2015 / \mathrm{CG} / 02$ for supporting this work.

\section{References:}

1. Clapham H.E., Tricou V., Chau N.V.V., Simmons C.P., Ferguson N.M. (2014). Within-host viral dynamics of dengue serotype 1 infection. J.R. Soc. Interface, 11:20140094. doi: 10.1098/rsif.2014.0094

2. Clark G., Gubler D. Dengue Fever, CDC Traveler's Information on Dengue Fever. Centers for Disease Control, Atlanta, Ga, USA. Retrieved from www.cdc.gov.

3. Derouich M., Boutayeb A. (2006). Dengue Fever: Mathematical Modeling and Computer Simulation. Applied Mathematics and Computation, 177, 528-544. doi:10.1016/j.amc.2005.11.031 
4. Epidemiology Unit Ministry of Health Sri Lanka. National Plan of Action for Prevention and Control of Dengue Fever 2005-2009.

5. Gujarati T.P., Ambika G. (2014). Virus Antibody Dynamics in Primary and Secondary Dengue Infections. J. Math. Biol., 69(6-7), 1773-800. doi:10.1007/s00285-013-0749-4

6. Guzmán M.G., Kourí G. (2004). Dengue, one of the great emerging health challenges of the 21st century. Expert Review of Vaccines, vol. 3, no. 5, 511-520. doi:10.1586/14760584.3.5.511

7. Host Response to the Dengue Virus. Retrieved from

8. http://www.nature.com/scitable/topicpage/host-response-to-thedengue-virus-22402106.

9. Janeway CA, Jr, Travers P., Walport M., Shlomchik MJ (2001). Immunobiology: The Immune System in Health and Disease 5th edition. Garland Science, New York.

10. Kajiwara T.,Sasaki T. (2010). Global stability of pathogen-immune dynamics with absorption. Journal of Biological Dynamics, 4:3, 258269. doi:10.1080/17513750903051989

11. Murphy B.R., Whitehead S.S. (2011). Immune Response to Dengue Virus and Prospects for a Vaccine. Annual Review of Immunology, 29,587-619.

12. Nikin-Beers R.P., Ciupe S.M. (2015). The role of antibody in enhancing dengue virus infection. Mathematical Biosciences, 263(may 2015), 83-92. doi: 10.1016/j.mbs.2015.02.004

13. Nowak M.A., May R.M. (2000). Virus Dynamics: Mathematical Principles of Immunology and Virology, Oxford University Press.

14. Nuraini N., Tasman H., Soewono E., Sidarto K.A. (2009). A with-in host dengue infection model with immune response. Mathematical and Computer Modeling, 49: 1148-1155. doi:10.1016/j.mcm.2008.06.016

15. PAHO (1994). Dengue and dengue hemorrhagic fever in the Americas: Guidelines for prevention and control, Scientific Publication No. 548, Pan American Health Organization.

16. Perera S.D., Perera S.S.N., Jayasinghe S. (2016). Modeling and Sensitivity of Dengue Viral Dynamics. International Journal of Current Research, 8(7), 34899-34906

17. Pradeep B.G.S.A., Ma W., Guo S. (2015). Stability Properties of a Delayed HIV Model with Nonlinear Functional Response and Absorption Effect. Journal of National Science Foundation, 43 (3), 235-244 (SCI)

18. Rodrigues H.S., Monteiro M.T.T., Torres D.F.M. (2012). Modeling and Optimal Control Applied to a Vector Borne Disease. 
International Conference on Computational and Mathematical Methods in Science and Engineering, Vol.3, 1063-1070.

19. Rodrigues H.S., Monteiro M.T.T., Torres D.F.M. (2013). Sensitivity Analysis in a Dengue Epidemiological Model. Conference Papers in Mathematics, Vol.2013, Art ID.721406. doi: 10.1155/2013/721406

20. Rotem B., Koelle k. (2014). Minimal within-host dengue models highlight the specific roles of the immune response in primary and secondary dengue infections. J.R. Soc. Interface, 12:20140886. doi:10.1098/rsif.2014.0886

21. Sirisena P.D.N.N., Noordeen F. (2014). Evolution of dengue in Sri Lanka-changes in the virus, vector, and climate. International Journal of Infectious Diseases, Vol. 19, 6-12.

22. Summer T. (2010). Sensitivity Analysis in Systems Biology Modeling and its Application to a Multi-Scale Model of Blood Glucose Homeostasis. PhD Thesis, Centre for Mathematics and Physics in the Life Sciences and Experimental Biology, University College London.

23. Wahala M.P.B., de Silva A.M. (2011). The Human Antibody Response to Dengue Virus Infection. Viruses, 3: 2374-2395. doi: 10.3390/v3122374

24. WHO-VMI Dengue Vaccine Modeling Group (2012). Assessing the Potential of a Candidate Dengue Vaccine with Mathematical Modeling, PLOS Neglected Tropical Diseases 6(3): e1450 